\title{
From force chains to nonclassical nonlinear dynamics in cemented granular materials
}

\author{
T. J. Ulrich $\odot^{*}$ \\ Detonation Science and Technology (Q-6), Los Alamos National Laboratory, Los Alamos, New Mexico 87545, USA \\ Zhou Lei, Marcel C. Remillieux, Esteban Rougier @, and Harvey E. Goodman (1) \\ Geophysics Group (EES-17), Los Alamos National Laboratory, Los Alamos, New Mexico 87545, USA \\ Katelyn A. Huffman \\ Chevron Energy Technology Company, Houston, Texas 77002-7327, USA \\ Peter Connolly 10 \\ ConnollyGeo, Houston, Texas 77019-4334, USA
}

(Received 2 November 2021; accepted 18 January 2022; published 10 February 2022)

\begin{abstract}
In this letter, we present evidence for a mechanism responsible for the nonclassical nonlinear dynamics observed in many cemented granular materials that are generally classified as mesoscopic nonlinear elastic materials. We demonstrate numerically that force chains are created within the complex grain-pore network of these materials when subjected to dynamic loading. The interface properties between grains along with the sharp and localized increase of the stress occurring at the grain-grain contacts leads to a reversible decrease of the elastic properties at macroscopic scale and peculiar effects on the propagation of elastic waves when grain boundary properties are appropriately considered. These effects are observed for relatively small amplitudes of the elastic waves, i.e., within tens of microstrain, and relatively large wavelengths, i.e., orders of magnitude larger than the material constituents. The mechanics are investigated numerically using the hybrid finite-discreteelement method and match those observed experimentally using nonlinear resonant ultrasound spectroscopy.
\end{abstract}

DOI: 10.1103/PhysRevE.105.L022901

The peculiar dynamic behavior of cemented granular materials finds its origin in their complex mesoscopic structure, which is essentially a disordered network of hard elements (e.g., quartz grains with characteristic lengths ranging from tens to hundreds of microns) cemented by soft bonds at the grain-grain interfaces and may be surrounded by an interconnected network of pores [1]. The pore space can host some fluid and add complexity to its structural response. Many independent experiments have revealed that these materials exhibit properties related to nonlinear and nonequilibrium dynamics, including the dependence of elastic parameters and attenuation on strain amplitude, slow dynamics, and hysteresis with end-point memory [2-4]. These properties have important implications for many applications ranging from structural integrity in civil engineering to oil and gas exploration in geophysics [5], as well as performance of polycrystalline energetic materials [6].

The study described herein compares experimentally obtained results from a cemented granular material (Berea

\footnotetext{
${ }^{*}$ Correspondence author: tju@lanl.gov

Published by the American Physical Society under the terms of the Creative Commons Attribution 4.0 International license. Further distribution of this work must maintain attribution to the author(s) and the published article's title, journal citation, and DOI.
}

sandstone) using nonlinear resonant ultrasound spectroscopy (NRUS) with numerical simulations of the same material and dynamic loading using a combined finite-discrete element method (FDEM) in an effort to gain micro- and mesoscopic understanding of possible mechanisms for the observed hysteretic nonlinear elastic behavior. FDEM is an effective tool for addressing a variety of physics problems formulated not only in terms of the continuum assumption and differential equations but also in terms of a large number of discrete entities interacting with one another [7,8]. Specifically, FDEM merges the advantages of the finite-element methods (FEM) for modeling material deformation and the strengths of the discrete-element methods for handling interparticle interactions [8-10]. As a result, the details of the focused physical system, such as the microscale structure of the cemented granular materials, the mechanical response of the grains, and laws governing interparticle interactions, can be explicitly represented and modeled via FDEM, thus the number of assumptions is minimized.

While the NRUS experiments were conducted on a solid cylinder $(l=30 \mathrm{~cm}, d=2.5 \mathrm{~cm})$ of the material, computational restrictions limited the simulations to two-dimensional (2D) cross sections at smaller length scales $(1 \mathrm{~mm} \times 1 \mathrm{~mm}$ and $2 \mathrm{~mm} \times 18 \mathrm{~mm}$ ). As the geometric dimensions dictate the resonance frequencies, it is necessary to scale the experimental and simulation results to facilitate comparison. The resonance frequency of a sample is directly proportional to its 

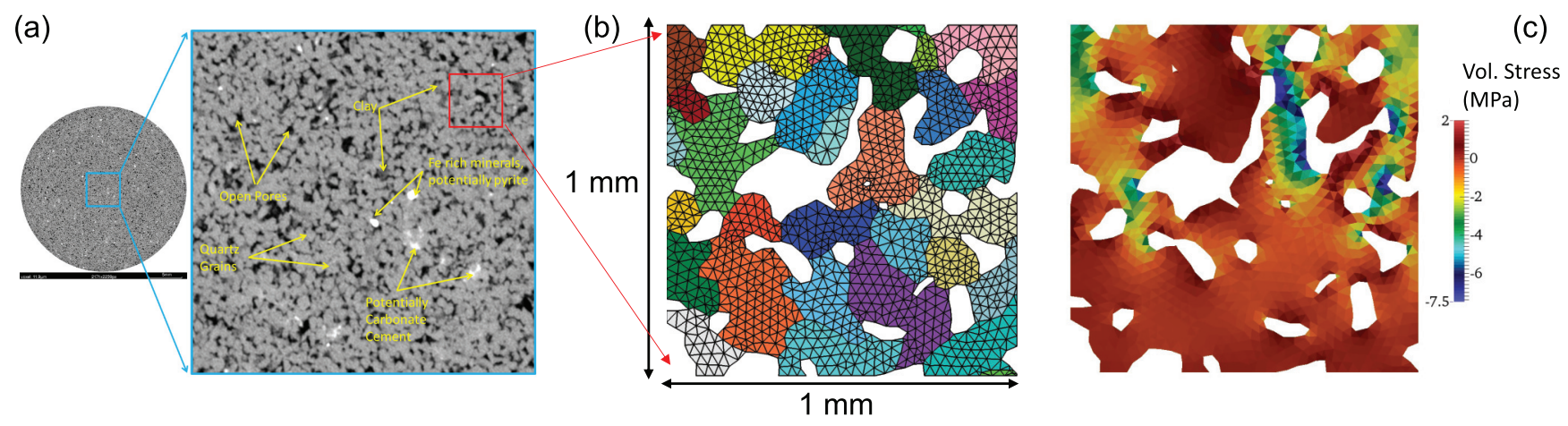

FIG. 1. (a) A cross section of the $\mathrm{x}$-ray CT measurement with materials and pore spaces identified; (b) a subset $(1 \mathrm{~mm} \times 1 \mathrm{~mm})$ of the $\mathrm{x}$-ray CT data colored to identify individual grains and meshed for FDEM and FEM simulations; (c) FDEM results (volumetric stress) from a uniaxially applied cyclic load (aligned vertically to the image) illustrating localized stress concentrations at grain-grain boundaries. Note: The highlighted regions [i.e., blue and red boxes in (a)] are for illustrative purposes only and thus are not to scale, nor do they represent the exact subset of grains used.

length, thus the scaling was done by normalizing the observed resonance frequency shifts $(\Delta f)$ by the lowest-amplitude resonance frequency $\left(f_{0}\right)$ for each system. Also, the loss of a dimension from 3D (experiment) to 2D (simulations) means that precise quantifications are likely not entirely relevant, and thus we will be restricted to qualitative comparisons where necessary.

The NRUS study presented here was restricted to longitudinal modes of vibration, the experimental details for which, as well as the sample details, have been reported previously and can be found in Refs. [11-13]. A high-resolution x-ray computed tomography (CT) image of the entire sample was also obtained, providing a visualization of the grain and pore structure of the cemented granular material with a spatial resolution of $10 \mu \mathrm{m}$. Small cross sections of the CT reconstruction were extracted and used to define meshes for the simulations presented here. Examples of the CT reconstruction and extracted cross sections can be seen in Fig. 1.

From the cross sections, we can see that the sample is composed of an ensemble of grains that are bonded to one another via thin cementitious layers. Each grain can be considered as a deformable discrete particle that interacts with its neighbors when under stress. In order to fully capture the dynamic response of the system, the numerical methods used for this purpose should (a) describe the evolution of the stresses as a function of the deformation inside each grain and (b) capture the grain-to-grain interaction physics and its effects on the overall response of the sample. Here we apply the FEM and the aforementioned hybrid FDEM to investigate a nonlinear elastic softening and determine the importance of grain-grain interactions as its origin.

For both the FEM and FDEM simulations, the same mesh definitions were used to discretize the grains. In both cases, the deformation of the grains is described using a multiplicative decomposition-based formulation [10]. The only difference between FEM and FDEM simulations is due to the fact that the thin cementitious layers between grains are not captured in the FEM models, while the mechanical response of the cementitious layers is modeled explicitly through the interface elements in FDEM, see Fig. 2. The interfaces between grains are assumed to have zero initial thickness and to coincide with the boundary of the grains defined through the finite-element mesh, i.e., grain boundary lines in Fig. 1(b). The relative displacement between any two adjacent interface surfaces is calculated based on the geometry of the corresponding grains, as shown in Fig. 2. The stresses in the bonds along the thin cementitious layer are then defined as functions of the relative displacement [14].

The material of the grains was assumed to be quartz with a Young's modulus of 97.8 GPa, a Poisson's ratio of 0.007 , and a density of $2650 \mathrm{~kg} / \mathrm{m}^{3}$ [15]. In this work, since the strain $(\epsilon)$ amplitudes of the loads are sufficiently small $(\sim 2-50 \mu \epsilon$, i.e., $\delta<<\delta_{t}$, see Fig. 2), an elastic material model was used to describe the stresses as a function of the deformation [10]. The thin layer of cementitious material was assumed to connect

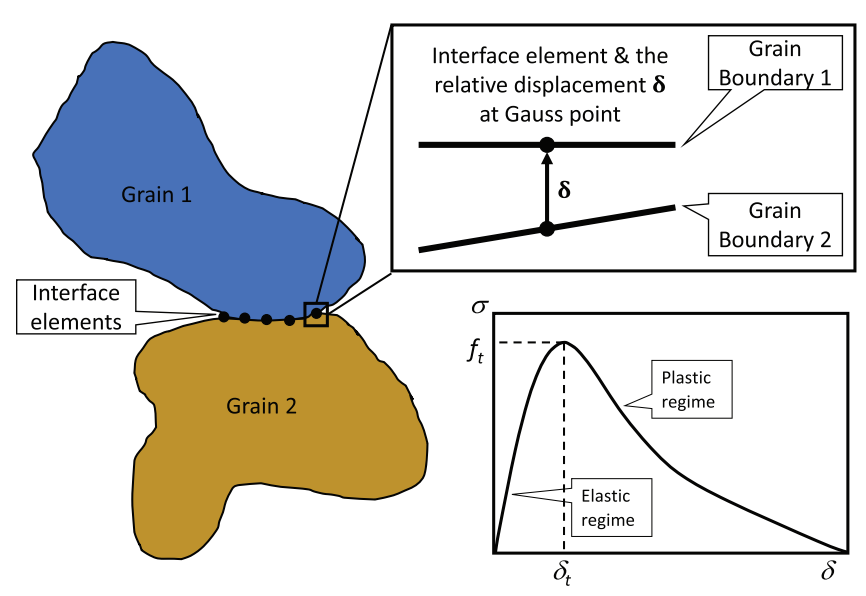

FIG. 2. The thin cementitious layers is represented through the interface elements in the FDEM models. The stresses are calculated at integration points (i.e., Gauss points) along the interface. The lower right plot shows a schematic representation of a typical curve used to define the material constitutive relationship between bonding stress $(\sigma)$ and relative displacement $(\delta)$. The strength of the material and the associated relative displacement are denoted by $f_{t}$ and $\delta_{t}$, respectively. It is important to note that this discontinuous feature is unique to the FDEM simulations and is not considered by continuum-based FEM models. 

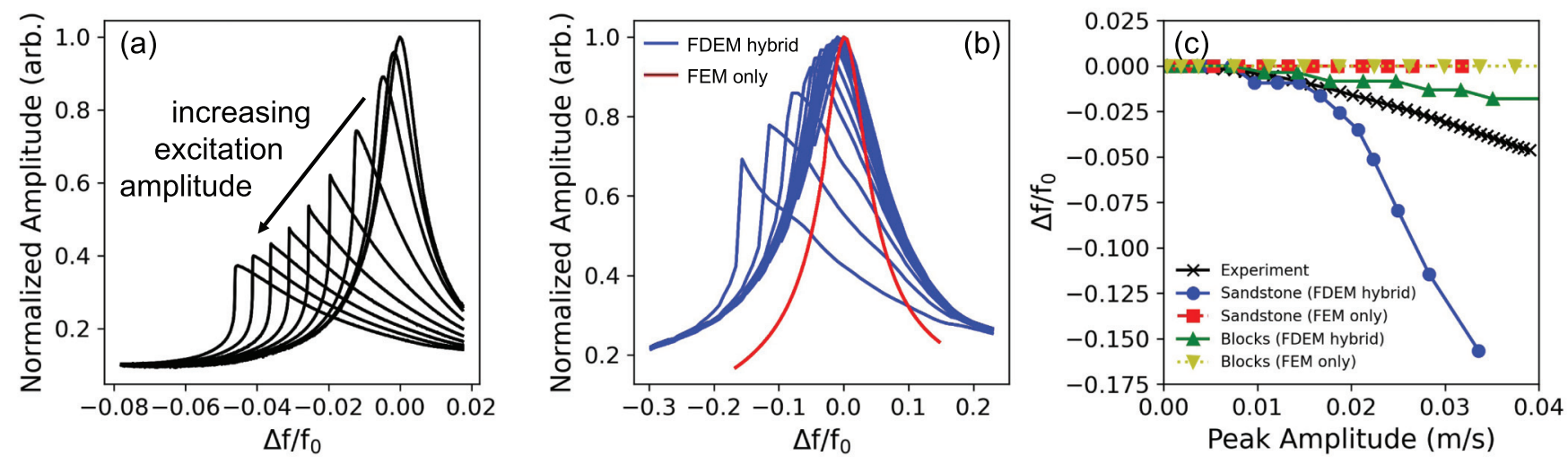

FIG. 3. NRUS results comparing (a) experiment to (b) $2 \mathrm{~mm} \times 18 \mathrm{~mm}$ simulations. The peak amplitudes have been normalized using excitation amplitude and lowest peak amplitude in order to visualize the nonlinear attenuation in addition to the peak shift. Frequency shift is reported as a fraction of the lowest-amplitude resonance frequency. Ten resonance amplitudes are shown from the experimental data and 13 amplitudes from both the FDEM and FEM simulations, respectively. (c) Relative peak shift as a function of peak amplitude, reported as particle velocity $(\mathrm{m} / \mathrm{s})$, for all simulations and the experiment.

(i.e., bond) each grain to its neighbors. This cementitious layer is defined by soft elastic moduli (i.e., $20 \mathrm{GPa}$ ) compared to the grains, low ultimate strengths (i.e., a tensile strength of $2 \mathrm{MPa}$ and a shear strength of $6 \mathrm{MPa}$ ), and a frictional parameter (i.e., a Coulomb friction coefficient of 0.7 ). The pore space, shown as white in Fig. 1(c), is empty with free boundary conditions. The material and interface parameters were used consistently across all simulations. The ability to allow for fracture and/or grain sliding, which involves the ultimate strength and frictional parameters, respectively mentioned above, can be selectively enabled or disabled in the simulations. At the small strains $\left(10^{-7}<\epsilon<10^{-4}\right)$ considered here, the effects of damage accumulation and friction were minimal, expressed as perturbations around the results of the FDEM simulations presented herein.

Three different grain arrangements were used for the simulations. As indicated, 2D cross sections of $1 \mathrm{~mm} \times 1 \mathrm{~mm}$ and $2 \mathrm{~mm} \times 18 \mathrm{~mm}$ were taken from the CT images. The grains were meshed according to their size and the interfaces defined at grain-grain contacts. Additionally, a quasi-1D rectangular series of grains with no porosity was constructed. The quasi1D geometry consisted of 120 rectangular blocks, $0.05 \mathrm{~mm}$ $\times 0.15 \mathrm{~mm}$ each, aligned along the short end to produce a $0.05 \mathrm{~mm} \times 18 \mathrm{~mm}$ chain of rectangular grains with flat interfaces. The latter geometry was formed to separate effects arising from geometric stress concentrations. In addition to FDEM simulations performed on all of the mesh geometries, FEM simulations were conducted on the $2 \mathrm{~mm} \times 18 \mathrm{~mm}$ and quasi-1D models. The FEM simulations do not support intergranular physics and thus treat the entire mesh area as a single piece of material. The rectangular vs. arbitrary grain shape and FDEM vs. FEM simulations allows for the investigation of nonlinear effects originating from geometric stress concentrations, e.g., Hertzian contact type nonlinearities, vs. interfacial physics, e.g., grain sliding and reorientation.

Both the NRUS experiments and simulations were conducted in the same modality, i.e., achieving a steady-state response to a constant amplitude, single-frequency sinusoidal excitation at one end of the sample and recording the response at the opposite end; stepwise increasing the excitation frequency to construct a resonance curve. This process is repeated with stepwise increased amplitudes of the excitation sinusoids in order to construct a series of resonance curves at defined strain amplitudes. The boundary conditions (BC's) of the experiment and the nonexcitation boundaries of the simulations were free. In linear systems, the resonance frequencies are independent of the excitation amplitude, while nonlinear resonant systems display an amplitude-dependent resonance frequency shift. In cemented granular material this is typically seen as a downward frequency shift, indicating an elastic softening with increased strain. This softening can be quantified by the third-order elastic constant, $\alpha$, from the proportionality of the relative shift to the strain amplitude. The results presented in Fig. 3 illustrate the typical experimental observations along with the results from each of the FDEM and FEM simulations. Accompanying the frequency shift, an amplitude-dependent attenuation manifests as a straindependent loss of efficiency in driving the resonance. This can be readily seen by normalizing the resonance curves by their corresponding drive amplitudes, as the NRUS data are presented in Fig. 3.

The experimental results shown in Fig. 3 illustrate both nonlinear effects, i.e., nonlinear attenuation and frequency shift, as do the FDEM simulation results presented in the same figure. It is apparent that when the interfacial mechanics are removed, as in the FEM simulations, these nonlinear effects disappear entirely, observed as the complete overlapping of the normalized resonance peaks. Qualitatively, we see identical behavior for all investigated systems where interface micromechanics are in play, including the experimental data. The peak shift as a function of the resonance-peak amplitude is presented in Fig. 3 for each of the cases. The horizontal lines for the FEM simulations reiterate the lack of amplitude dependence of resonance frequency, thus indicating no hysteretic nonlinearity is present without the addition of the interfacial physics. The amplitude dependence of resonance frequencies for all FDEM simulations and the experimental data exhibit the same type of response, though to varying degrees.

The FDEM simulations provide the ability to visualize the internal mechanics of the granular medium, which can 

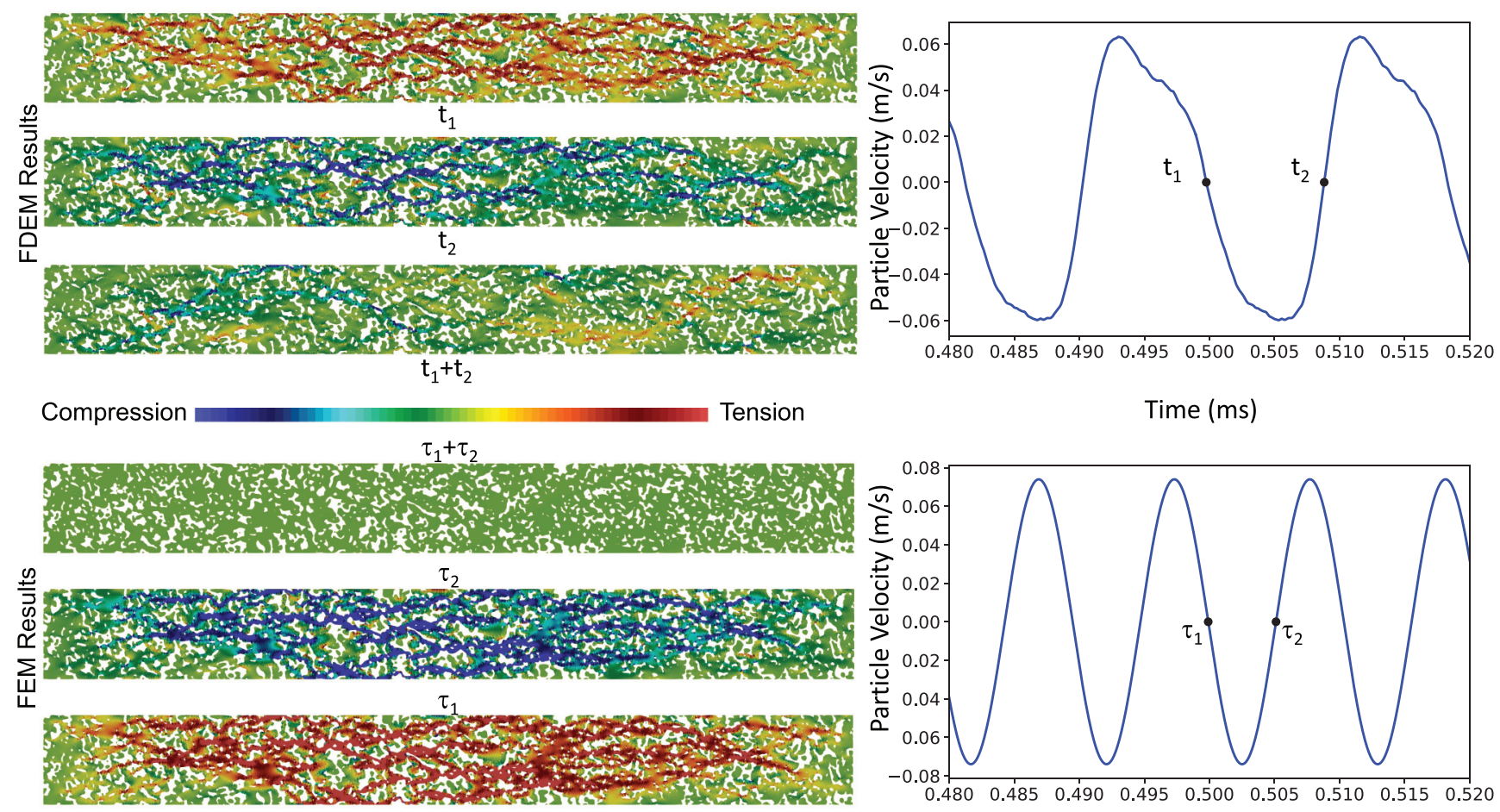

FIG. 4. Force chains, from FDEM (left upper half) and FEM (left lower half) simulations, for the lowest-order resonance mode for the sandstone simulated cross section $(2 \mathrm{~mm} \times 18 \mathrm{~mm})$. Force chains are shown for the extremes of the resonant mode (i.e., tension and compression) at times $t_{1}$ and $t_{2}\left(\tau_{1}\right.$ and $\tau_{2}$ for FEM), respectively, as illustrated in the waveform plots (right). Notice the difference in the force chains from tension to compression $\left(t_{1}+t_{2}\right)$ for the FDEM simulations where interface mechanics are enabled, as opposed to the identical force chains seen from analogous FEM simulations.

be seen in Fig. 4 as the formation of force chains, or stress percolation, in a similar manner as seen in FEM simulations of granitic rocks by Burnley [16]. From these we can gain visual insight into the mechanics of the force chains at different moments of the loading cycle. When comparing the sample structure, specifically the volumetric stress, it becomes apparent that the force chains at the moment of maximum tension $\left(t_{1}\right)$ are slightly different than those present at the moment of maximum compression $\left(t_{2}\right)$. This can be observed in Fig. 4 when summing the maximal force chain images at $t_{1}$ and $t_{2}$. With this change in load bearing structure throughout the transition from tension to compression, and the reverse, it is of no surprise that the elastic response would exhibit hysteretic behavior. In contrast, the FEM simulations of the same structure qualitatively form a similar set of force chains (Fig. 4, lower half); however, when comparing those force chains at maximum tension and compression, they are identical (Fig. 4, $\tau_{1}+\tau_{2}$ panel). The lack of a hysteretic nonlinear elastic response in the FEM simulations is thus consistent with unchanging force chains when loading or unloading and highlights the importance of capturing the interfacial physics using FDEM, further supporting the connection between nonlinear hysteretic elasticity and hard-soft force chains.

In conclusion, a possible mechanism of interface mechanics in force chains has been identified that can result in a nonlinear dynamic response as observed in cemented granular media. This is consistent with past descriptions of nonlinear mesoscopic elastic materials being hard elements with soft contacts, as well as with past experimental work by Darling et al. [17], which used neutron measurements to isolate the mechanism to grain boundaries, though were not able to provide insight or visualization into the mesomechanical behavior of the force chains. The model used here to simulate and explore this mechanism utilizes simple constitutive laws of mechanics that give rise to the expected nonlinear behavior through the complexity of the system's structure rather than through ad hoc phenomenological schemes. In the end, first-principles-based FDEM simulations demonstrate that soft interface properties in granular chains do indeed give rise to nonlinear hysteretic elastic behavior. Connecting nonclassical nonlinear, i.e., hysteretic, elasticity to the fundamental mechanics incorporated into FDEM opens new doors to the understanding, prediction, and utilization of nonlinear elastic phenomena.

It should be reiterated that the studies included in this communication focused on dry systems, i.e., absent any pore fluid. This is strictly true for all of the simulations, where fluids can be eliminated completely, while the experiments on the Berea sandstone were conducted in room-dry conditions at the Los Alamos National Laboratory (LANL). With that in mind, we observe consistent nonlinear elastic behavior in both the simulations and experiments without the need to employ mechanisms involving fluids and fluid-solid interactions. The authors, however, do acknowledge that fluid-based mechanisms may also alter the nonlinear elastic behavior 
[18] and possibly even create nonlinear behavior in otherwise linear systems. The FDEM software, specifically the LANL developed Hybrid Optimization Software Suite [19], used to conduct the simulations here, includes a fluid-solid interaction solver, and thus future studies could expand on those presented here and include fluid effects.
The data that support the findings of this study are available from Chevron Energy Technology Company. Restrictions apply to the availability of these data, which were used under license for this study. Data are available from the authors on reasonable request and with the permission of Chevron Energy Technology Company.
[1] R. A. Guyer and P. A. Johnson, Phys. Today 52(4), 30 (1999).

[2] L. Ostrovsky and P. Johnson, Riv. Nuovo Cimento Soc. Ital. Fis. 24, 1 (2001).

[3] J. A. TenCate, E. Smith, and R. A. Guyer, Phys. Rev. Lett. 85, 1020 (2000).

[4] K. Winkler, A. Nur, and M. Gladwin, Nature (Lond.) 277, 528 (1979).

[5] J. A. TenCate and M. Remillieux, Acoust. Today 15, 29 (2019).

[6] P. Schulze, E. Pittman, S. Sjue, D. S. Moore, T. Feagin, and C. Donahue, Propell. Explos. Pyrotechn. 45, 387 (2020).

[7] A. Munjiza, Discrete elements in transient dynamics of fractured media, Ph.D. thesis, Swansea University, 1992.

[8] A. Munjiza, The Combined Finite-Discrete Element Method, 1st ed. (John Wiley \& Sons, Chichester, UK, 2004).

[9] A. Munjiza, E. E. Knight, and E. Rougier, Computational Mechanics of Discontinua, 1st ed. (John Wiley \& Sons, Chichester, UK, 2011).

[10] A. Munjiza, E. Rougier, and E. E. Knight, Large Strain Finite Element Method: A Practical Course, 1st ed. (John Wiley \& Sons, Chichester, UK, 2015).
[11] M. C. Remillieux, R. A. Guyer, C. Payan, and T. J. Ulrich, Phys. Rev. Lett. 116, 115501 (2016).

[12] M. C. Remillieux, T. J. Ulrich, H. E. Goodman, and J. A. Ten Cate, J. Geophys. Res.: Solid Earth 122, 8892 (2017).

[13] M. Lott, M. C. Remillieux, V. Garnier, P.-Y. Le Bas, T. J. Ulrich, and C. Payan, Phys. Rev. Materials 1, 023603 (2017).

[14] Z. Lei, E. Rougier, E. E. Knight, M. Zang, and A. Munjiza, Appl. Sc. 11, 2484 (2021).

[15] M. W. Lee, Proposed Moduli of Dry Rock and Their Application to Predicting Elastic Velocities of Sandstones, Technical Report 2005-5119 (USGS Publications Warehouse, 2005).

[16] P. C. Burnley, Nat. Commun. 4, 2117 (2013).

[17] T. W. Darling, J. A. TenCate, D. W. Brown, B. Clausen, and S. C. Vogel, Geophys. Res. Lett. 31, L16604 (2004).

[18] J. A. Bittner and J. S. Popovics, Appl. Phys. Lett. 114, 021901 (2019).

[19] E. E. Knight, E. Rougier, Z. Lei, B. Euser, V. Chau, S. H. Boyce, K. Gao, K. Okubo, and M. Froment, Comput. Particle Mech. 7, 765 (2020). 